\title{
The Influence of Heart and Lung Dynamics on the Impedance Cardiogram — A Simulative Analysis
}

\author{
M. Ulbrich, A. Schauermann, S. Leonhardt
}

\begin{abstract}
Impedance cardiography (ICG) is a simple and cheap method for acquiring hemodynamic parameters. Unfortunately, not all physiological influences on the ICG signal have yet been identified. In this work, the influence of heart and lung dynamics is analyzed using a simplified model of the human thorax with high temporal resolution. Simulations are conducted using the finite integration technique (FIT) with temporal resolution of $103 \mathrm{~Hz}$. It is shown that changes in heart volume as well as conductivity changes of the lung have a high impact on the ICG signal, if analyzed separately. Considering the sum signal of both physiological sources, it can be shown that they compensate each other and thus do not contribute to the signal. This finding supports Kubicek's model.
\end{abstract}

Keywords: impedance cardiography, bioimpedance, simulation, finite integration technique, high temporal resolution, signal source analysis.

\section{Introduction}

One of the most common causes of death in Western Europe is chronic heart failure (CHF). Measures of the severity of this cardiovascular disease are hemodynamic parameters such as stroke volume (SV). Until now, the gold standard for measuring these parameters has been the thermodilution technique, which utilizes a pulmonary artery catheter. However, risks of estimating $\mathrm{CO}$ via catheters include infections, sepsis and arrhythmias, as well as increased morbidity and mortality.

An alternative method for assessing hemodynamic parameters non-invasively and cost-effectively is ICG. Currently, ICG is not commonly used as a diagnostic method, because it is not considered to be valid [1]. One reason is the inaccuracy of the technology itself concerning SV calculations. Another possible reason for this is that processes in the human body during ICG measurements are widely unknown. One way to analyze where the current paths run and which tissue contributes significantly to the measurement result is to use computer simulations employing FIT. Other researchers have already examined multiple sources of the ICG signal, using various approaches: some works are based on simple geometries [2], others on real anatomical data, such as MRI data [3].

Since controversial results have been obtained, the influence of two particular sources for the impedance cardiogram will be analyzed in this work and will be judged according to the value of their contribution.

\section{Basics}

The basics of the measurement technique and also the simulation technique will be explained in this section.

\subsection{Bioimpedance}

For bioimpedance measurements, two outer electrodes are used to inject a small alternating current into the human body, and the voltage is measured by two inner electrodes to calculate the complex impedance. If a frequency spectrum between $5 \mathrm{kHz}$ and $1 \mathrm{MHz}$ is used to measure the bioimpedance for each frequency, this method is called bioimpedance spectroscopy (BIS). This is generally the most interesting frequency range for diagnosis, since physiological and pathophysiological processes lead to changes in body impedances with high dynamics. BIS is commonly used to assess the body composition of humans.

If only one frequency of this spectrum is used to measure the bioimpedance continuously, this method is called impedance cardiography (ICG). Using ICG, time-dependent hemodynamic parameters can be extracted from the measured impedance curve (see Figure 1). The derivation of the impedance change $\Delta Z$ is the ICG signal whose maximum is used for calculating the stroke volumes. The measured SV by ICG according to Bernstein and Sramek can be described by the following equation:

$$
S V=\delta \cdot \frac{(0,17)^{3}}{4,2} \cdot\left|\frac{\mathrm{d} Z}{\mathrm{~d} t}\right|_{\max } \cdot \frac{t_{e}}{Z_{0}}
$$




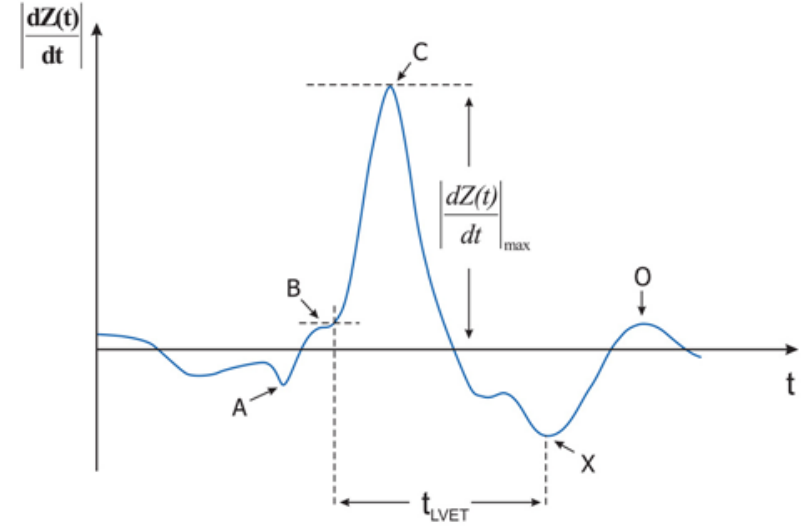

Fig. 1: Typical ICG Wave

Here factor $\delta$ is the actual weight divided by the ideal weight, $t_{e}$ is the left ventricular ejection time (LVET), and $Z_{0}$ is the thoracic base impedance [4]. Since ICG usually operates at one certain frequency between 20 and $100 \mathrm{kHz}$, only one continuous point on a complex frequency locus plot is obtained by ICG measurements.

\section{$2.2 \quad$ Finite Element Simulation}

Finite Element simulations may be used to describe complex structures by subdividing them into simple finite elements. These elements can be triangles or rectangles for $2 \mathrm{D}$ problems, and tetrahedrons, pentahedrons, pyramids or hexahedrons for 3D problems. Discretization using hexahedrons is shown in Figure 2 (middle). Physical values are assigned to the edges, faces and volumes of these substructures. This assignment is illustrated in the following figure (right), which shows 2 dual grids representing the functional principle of the FIT.

Here, electric voltages (e) and magnetic voltages (h) are assigned to the edges while the magnetic fluxes (b) and electric fluxes $(\mathrm{j}, \mathrm{d})$ are allocated on the faces of the grids. Hence, a system of equations, the Maxwell-Grid-Equations, has to be solved for the whole calculation domain describing each cell:

$$
\begin{array}{ll}
C \vec{e}=-\frac{\partial \vec{b}}{\partial t} & \tilde{C} \vec{h}=\frac{\partial \vec{d}}{\partial t}+\vec{j} \\
\tilde{S} \vec{d}=q & S \vec{b}=0
\end{array}
$$

Note that matrices $C$ and $\tilde{C}$ correspond to the curl operator, and matrices $S$ and $\tilde{S}$ correspond to the divergence operator [5]. While the differential form of Maxwell's Equations is solved for FEM simulations, the integral form is used for FIT simulations, so that the material matrices are diagonal for FIT while the FEM material matrices are non-diagonal. These matrices contain information about conductivity, permittivity and permeability, and define the relations between voltages and fluxes [6].

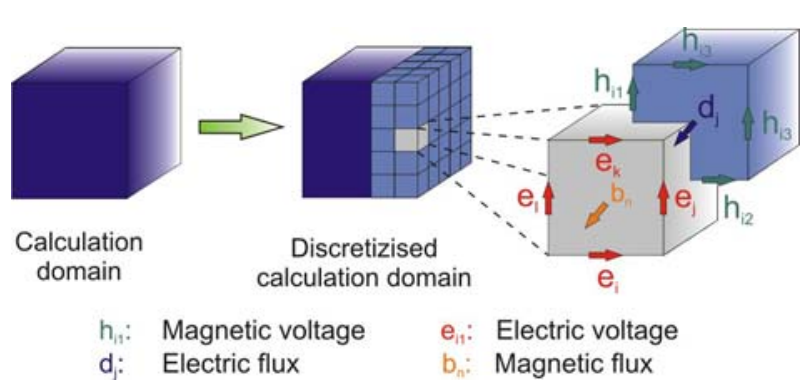

Fig. 2: Finite Integration Technique

\section{Methods}

Classical ICG analyzes the impedance of the thorax, approximating its volume by one outer cylinder with a conductivity from a mixture of tissues, containing another cylinder representing the aorta. This is of course an assumption which leads to modeling errors. As a result, the task is to find other models to improve the reliability of ICG measurements. In addition, it has to be clarified which physiological sources contribute to the ICG signal and lead to its characteristic points, shown in Figure 1.

This task will be accomplished by simulations using FIT and an anatomical data set of a male human as a basis for a simplified dynamic model. The dataset is based on the Visible Human Project $\left.{ }^{(}\right)$ dataset from the National Library of Medicine in Maryland [7]. Since this dataset contains no information about dynamics, a new model had to be created using simple geometries, such as frustums, spheres and cylinders, in order to reduce the simulation time. The thorax of the Visible Human Male in combination with the created simple thorax is shown in Figure 3 .

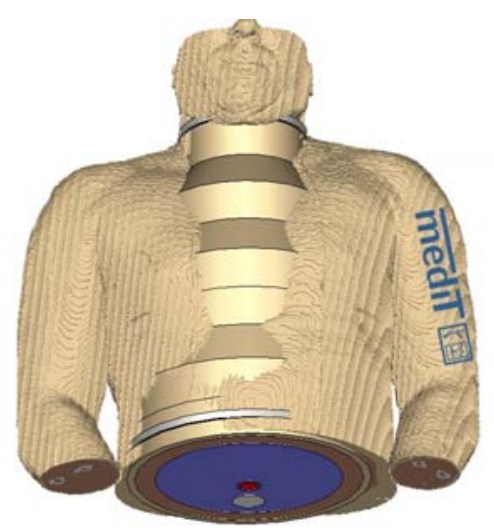

Fig. 3: Visible Human dataset and simplified model

For each expansion step a new model had to be created, since for each point in time the discretization of the simulated volume has to be recalculated due to the altered thorax geometry. The left ventricular volume change obtained by MRI data has been used to alter the radius of a sphere representing the 
heart while assuming a stroke volume of $80 \mathrm{ml}$ [8]. The radius varies between $38.5 \mathrm{~mm}$ and $33.8 \mathrm{~mm}$ and will mimic the contraction of the heart. The conductivity change due to lung perfusion has been implemented by assuming a maximum conductivity change of $10 \%$ [9]. Based on measured conductivity changes with electrical impedance tomographic spectroscopy (EITS), a dataset comprising 103 points in time has been created using Matlab [10].

The calculation frequency has been set to $100 \mathrm{kHz}$. Using a discretization density of 50 units, each model comprises 1.5 million tetrahedrons. Besides this global discretization, finer local discretization has been used for shape changing organs in order to obtain accurate results for small geometry changes. Conductivity $(\sigma)$ and permittivity $\left(\epsilon_{r}\right)$ values for every tissue have been implemented using the data from Gabriel et al. (see Table 1).

Table 1: Permittivity and conductivity values for $100 \mathrm{kHz}[11]$

\begin{tabular}{|l||l|c|}
\hline & $\sigma[\mathrm{S} / \mathrm{m}]$ & $\epsilon_{r}$ \\
\hline Blood & 0.70292 & 5120 \\
\hline Myocard & 0.21511 & 9845.8 \\
\hline Bone & 0.020791 & 227.64 \\
\hline Fat & 0.024414 & 92.885 \\
\hline Muscle & 0.36185 & 8089.2 \\
\hline Abdomen & 0.2 & 4000 \\
\hline Lung & 0.10735 & 2581.3 \\
\hline
\end{tabular}

Rebuilding all organs situated in the abdomen with the chosen procedure would have been too complex for the desired model. In addition, the impedance change of these organs plays a subordinate role for the ICG signal during a heartbeat, so that the simplification has been made that the abdominal part of the model has been filled with a uniform "tissue mixture" by using Boolean operations. Average permittivity and conductivity of all abdominal organs has then been assigned to this tissue. For simplicity, ring electrodes have been used for current injection. Of course, it is basically possible to use standard spot electrodes for the simulation.

The time resolution comprises 103 points in time within a heartbeat for both physiological sources. For validation purposes, the sum of the dynamic impedance measured at $100 \mathrm{kHz}$ and including the volume change of the aorta has been compared to measured data of a male human and the results show excellent agreement $(r=0.94)$ using a correction factor $[12]$. This data has been acquired using the Niccomo $^{\text {TM }}$ device from medis Germany, Ilmenau. Thus, the model itself has been considered to be suitable for these kind of simulations.

\section{Results}

The model created for the simulation is shown in Figure 4. It consists of all important tissues (muscle, fat, bone, heart, lung, abdominal tissue, important blood vessels). The current density results of a simulated measurement are presented here in a transversal section by arrows floating through the thorax from abdomen to neck. We can observe visually that high current density persists in muscle tissue, aorta and heart whereas low current flows through bone and fat tissue. It is also no surprise, that there is high current density in the current injection areas.

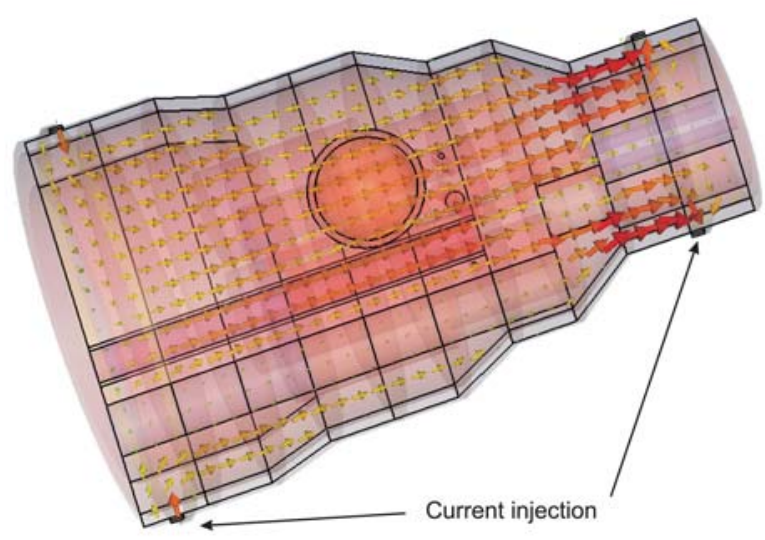

Fig. 4: Simulation model and current density results

All simulations have been calculated on a personal computer with a 64 bit operating system, an Intel ${ }^{\circledR}$ Xeon ${ }^{\circledR} 5240$ processor with 2 cores and 24 GB RAM. Every simulation step consumed 2 GB RAM and lasted 5 minutes using one core of the processor. Impedance changes have been computed for all points in time and both dynamic sources, so that in total 206 simulations have been conducted (see Figure 5).

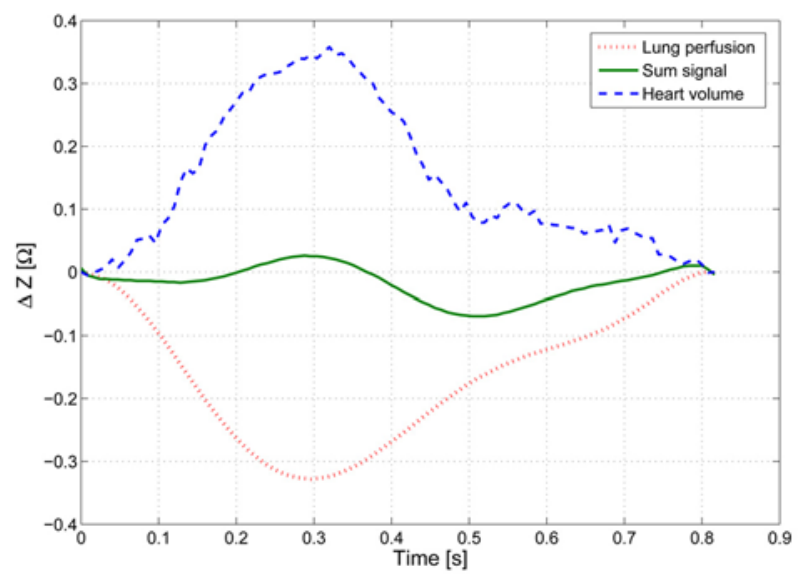

Fig. 5: Simulation results of one heartbeat 
This plot shows the results of lung perfusion simulations and heart beat simulations, as well as the sum signal of both results. The ripple of the heart impedance change can be explained by the changing geometry of the model due to the heart volume change, because for every new model geometry a new mesh has to be created. This can lead to other edges or faces of the grid contributing to the results obtained by the postprocessor.

What is more, an impedance change of approx. $0.35 \Omega$ can be observed for both dynamic sources. In addition, both results show nearly the same temporal behavior, so that the resulting curve reveals no significant impedance change.

\section{Discussion}

The task of this work was to analyze the influence of lung perfusion and heart beat on ICG measurements.

First, simulations with high temporal resolution have been conducted using conductivity changes of the lung and geometry changes of the heart as physiological dynamic sources for the ICG signal. In addition, physiological data and a simple model on the basis of anatomical data have been used to produce realistic results. Thus, a resource-saving and accurate way to reproduce an impedance measurement has been implemented. Second, it has been shown that lung perfusion and heartbeat have a high influence on the ICG signal because both signals have a maximum impedance change of $0.35 \Omega$, which is even higher than the usual impedance change of the thorax. Third, the two effects annihilate themselves because they show a very similar temporal behavior.

To sum up, it has been shown that although there are other big physiological influences altering the conductivity and thus the impedance of the human thorax, it seems that the original Kubicek model does not really make false assumptions by taking the aorta as the major contributor to the ICG signal into account and neglecting other sources.

This model has of course the potential to be improved. We could add more static sources, such as rib cage, kidneys and liver and more dynamic sources should be taken into account including erythrocyte orientation, respiration and more components of the cardiovascular system. In addition, the influence of pathologies on the ICG signal should be analyzed in order to explain effects when measuring patients.

\section{Acknowledgement}

The research described in this paper was supervised by Prof. Steffen Leonhardt, RWTH Aachen Uni- versity of Technology and was supported by Jens Mühlsteff, Philips Research Eindhoven. This work contributes to the European Union "HeartCycle" project.

\section{References}

[1] Cotter, G.: Impedance cardiography revisited, Physiological Measurement, 2006, vol. 27, p. $817-827$.

[2] Kosicki, J.: Contributions to the Impedance Cardiogram Waveform, Annals of Biomedical Engineering, 1986, vol. 14, p. 67-80.

[3] Patterson, R. P.: Sources of the thoracic cardiogenic electrical impedance signal as determined by a model, Medical \& Biological Engineering \& Computing, 1985, vol. 23, p. 411-417.

[4] Van De Water, J.: Impedance cardiography the next vital sign technology? Chest, 2003, vol. 123, p. 2028-2033.

[5] Clemens, M.: Discrete Electromagnetism with the Finite Integration Technique, Progress In Electromagnetics Research, 2001, p. 65-87.

[6] Demenko, A.: On the equivalence of Finite Element and Finite Integration formulations, 17th Conference on the Computation of Electromagnetic Fields, 2009, p. 677-678.

[7] National Library of Medicine, "The visible human project." [Online], Available: http://www.nlm.nih.gov/research/visible/

[8] Feng, W.: A Dual Propagation Contours Technique for Semi-Automated Assessment of Systolic and Diastolic Cardiac Function by CMR, Journal of Cardiovascular Magnetic Resonance, 2009, vol. 11.

[9] Brown, B. H.: Simultaneous Display of Lung Ventilation and Perfusion on a Real-Time Eit System, Engineering in Medicine and Biology Society, Proceedings of the Annual International Conference of the IEEE, 1992, vol. 5, p. 1710-1711.

[10] Zhao, T.: Modelling of Cardiac-Related Changes in Lung Resistivity Measured with EITS, Physiological Measurement, 1996, vol. 17, p. 227-234.

[11] Gabriel, C.: The dielectric properties of biological tissues, Physics in Medicine and Biology, 1996, vol. 41, pp. 2231-2 249. 
[12] Ulbrich, M.: Simulation of Continuous Spectroscopic Bioimpedance Measurements for Impedance Cardiography, IEEE International Workshop on Impedance Spectroscopy, 2010.

\section{About the authors}

Mark Ulbrich was born 1980 in Bonn, Germany. He studied electrical engineering at RWTH Aachen University of Technology and specialized in medical engineering. He started in 2009 working as Ph.D. student at the Chair for Medical Information Technology, RWTH Aachen University.

His research focuses on the implementation of a textile integrated bioimpedance measuring system which allows the monitoring of hemodynamic parameters at home.
Alexander Schauermann was born 1983 in Frunse (Kirghizia). He started his studies in electrical engineering at RWTH Aachen University in 2005.

Obtaining his bachelor degree in 2007, he focused his research interest on microelectronics and medical engineering during his master studies.

Mark Ulbrich

E-mail: ulbrich@hia.rwth-aachen.de

Alexander Schauermann

E-mail: alexander.schauermann@rwth-aachen.de Steffen Leonhardt

Philips Chair for Medical Information Technology

RWTH Aachen University

Pauwelsstrasse 20, 52074 Aachen, Germany 